\title{
Teaching and Learning through Laboratory Experiments in the Area of Nuclear Technology
}

Gustavo LAZARTE

Reactor Nuclear RA0, Facultad de Ciencias Exactas Fisicas y Naturales, Universidad Nacional de Cordoba, Argentina

Kouichi Julian Andres CRUZ

Reactor Nuclear RA0, Facultad de Ciencias Exactas Fisicas y Naturales, Universidad Nacional de Cordoba, Argentina

Alejandra Lucia PEREZ LUCERO

Reactor Nuclear RA0, Facultad de Ciencias Exactas Fisicas y Naturales, Universidad Nacional de Cordoba, Argentina

Norma Adriana CHAUTEMPS

Reactor Nuclear RA0, Facultad de Ciencias Exactas Fisicas y Naturales, Universidad Nacional de Cordoba, Argentina

Walter Miguel KEIL

Reactor Nuclear RA0, Facultad de Ciencias Exactas Fisicas y Naturales, Universidad Nacional de Cordoba, Argentina

\section{Abstract}

Simulated laboratories are an effective tool to complement teaching and learning processes, in this case, in the area of nuclear physics and related sciences. They can be used in universities, schools, and research centers for personnel ramp up and training. This work presents the development of a simulator of a nuclear radiation counter and the elements used in experiments alongside it, such as simulated radioactive sources, absorbing materials and dispersing materials of radiation. This simulator allows us to verify the scientific laws that are involved in the interaction of radiation with matter, in a safe and reproducible way. The simulated laboratory experiments include determining the plateau curve of a Geiger-Müller tube, beta particle absorption and backscattering, and radioactive background. The data obtained from the simulations is based on the real experiments, eliminating the inherent risks of the manipulation of radioactive materials. This also allows to verify theoretical concepts in practice, strengthening the learning process and incentivizing research, interpretation, 
integration and communication of the obtained results. By incorporating this simulator in the multidisciplinary teaching and learning processes in STEM fields, it is possible to run these laboratories in a simple manner using nonradioactive materials.

Keywords: teaching, learning, technology, experimentation, simulator, nuclear, simulated education

\section{Introduction}

Laboratory experimentation on Science, Technology, Engineering and Mathematics (STEM) subjects allows students, professors and professionals to apply interdisciplinary concepts where the theoretical aspects of scientific developments can be applied to a real-life activity. In these experiments, schools, scientific communities and the industry come together as a cohesive unit.

At the same time, the usage of simulators in teaching and personnel training is becoming wider in all stages of academic and/or professional career developments, especially on fields where the experiments are either complex, expensive or dangerous. Simulating these experiments transforms them into simple, cost-effective and safe experiments, through the use of the data obtained from real experiments. By doing this, the student can qualitatively and quantitatively verify the scientific laws that interact with the real world, in an environment with the aforementioned benefits (Perez Lucero et al., 2015).

Nuclear physics, in particular, is one of those fields. Most of the experiments involve the manipulation of radioactive material, which means that experiments must be done on nuclear laboratories with the proper protection to use them. This poses an accessibility problem for schools and universities who do not have a nuclear laboratory and want to teach about Nuclear Physics, as they would be unable to provide a valuable laboratory experimentation for their students.

In the upcoming sections, we present a project of a Geiger counter simulator, as a part of a broader project (Chautemps et al., 2019). This simulator puts the user in a similar environment as the real experiment environment, where the user can develop skills, learn operative procedures, and reinforce knowledge of theoretical and practical aspects from the real experiments. All of the simulations can be done an unlimited number of times, which also allows to adjust to the user's own learning process. The simulator presented in this work is the second iteration of said project, adding data for an additional experiment, and updating the user interface in order to simplify future upgrades, and extend the lifespan of the simulator. 


\section{Background}

Geiger counters are instruments used for detecting and measuring ionizing radiation. They consist of a Geiger-Müller tube, a cylinder filled with ionizing gas, and electrical components that count the amount of ionizing pulses that come from inside the tube. A basic scheme of the Geiger counter can be seen on figure 1 .

Whenever a charged particle (such as $\alpha$ or $\beta$ particles) goes into the detector, the gas gets ionized. This produces a chain reaction that lasts for a fraction of a second which, in turn, produces a pulse, which is processed by the pulse counter. The pulse occurs due to the power supply, as it forces electrons to accumulate on the anode, and positive ions on the cathode.

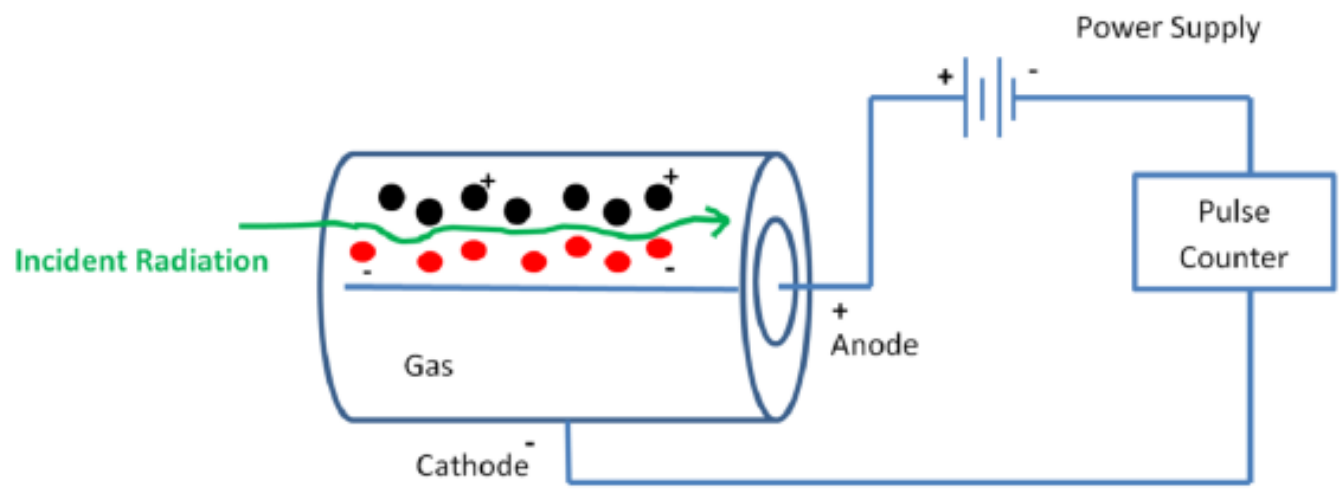

Figure 3: Geiger Counter Scheme

\section{Simulator description}

This section explains in detail the different laboratory experiments and how the user should operate the simulator in order to obtain the results from each experiment. The simulated elements include the electrical supply voltages of the Geiger counter, radioactive sources, absorbent elements and particle dispersers. These values are used to determine the Plateau curve of the aforementioned instrument, the values of the absorption and backscattering of charged particles by different materials exposed to the selected radioactive source, and the effects of background radiation.

This iteration of the simulator adds the capabilities for all measured data to variate according to known statistical distributions for each piece of data, unlike its predecessor which has fixed values for all data types (Lazarte et al., 2016). This variation of data allows the simulator to resemble the real experiment more closely, as there is always some degree of error and variation when measuring in the real-life experiment.

Laboratory 1: Determining the plateau curve of the Geiger Counter 
The plateau curve indicates the appropriate supply voltage for a Geiger counter. If the voltage is too low, radioactive activity will not be equally distributed along the cathode; if the voltage is too high, the tube may be damaged by the continuous discharges. An appropriate supply voltage will be in a zone from the curve in which the amount of counts per minute grows linearly when increasing voltage, with the optimal point being in the middle of this zone, to reduce the effect of variations in the voltage (Knoll, 2000). The graph shown in figure 2 shows a graphical representation of the plateau curve.

In order to experimentally obtain the aforementioned curve, the user would need to vary the supply voltages used on the tube, measuring on every variation the amount of counts per minute returned by the pulse counter. Using this data, the user will be able to plot the curve and graphically determine the optimal supply voltage.

In the simulator, the user is able to select the supply voltage from a set of pre-loaded data on the simulator, which has been obtained from doing the real experiment.

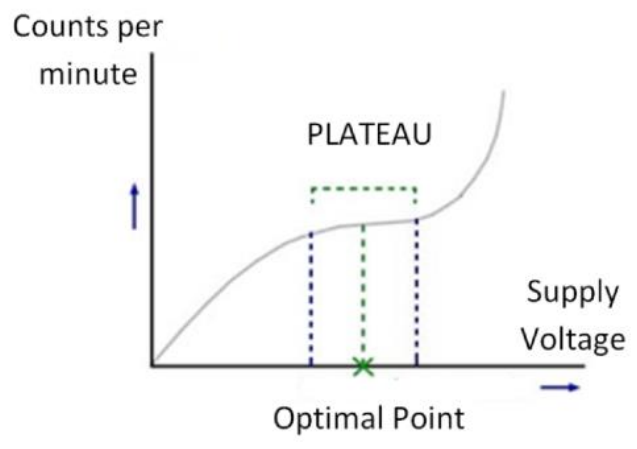

Figure 4: Plateau Curve of the Geiger Counter

Table 1 shows the measured activity (in counts per minute) as a function of the supply voltage of the detector. The values displayed on the simulator are slightly modified following a statistical normal distribution, in the same fashion as in the real experiment.

\begin{tabular}{|l|l|}
\hline V (voltage) & $\begin{array}{l}\text { Am - Background } \\
\text { (cpm) }\end{array}$ \\
\hline 550 & 0 \\
\hline 600 & 0 \\
\hline 625 & 0 \\
\hline 650 & 695 \\
\hline 675 & 1537 \\
\hline 700 & 1592.333 \\
\hline 725 & 1557.4 \\
\hline 750 & 1590 \\
\hline 775 & 1626 \\
\hline
\end{tabular}




\begin{tabular}{|l|l|}
\hline 825 & 1759.333 \\
\hline 850 & 2037.666 \\
\hline
\end{tabular}

\section{Table 2: Activity according voltage source}

The simulated elements are shown on figure 3.

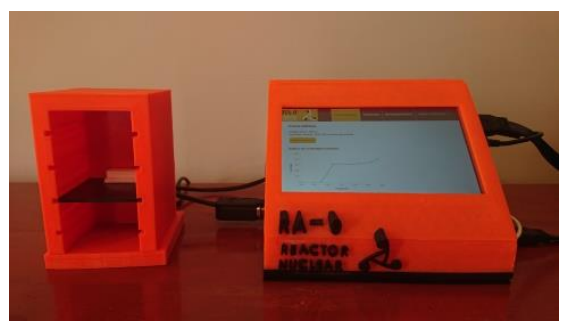

Figure 5: Simulated plateau curve of the Geiger counter

\section{Laboratory 2: Absorption}

The next laboratory focuses on two aspects of nuclear experimentation: on one hand, determining which radioactive material is acting as the radioactive source (that is, to determine whether the source is Cobalt-60, Sodium-22, Strontium-90, etc.); on the other hand, determining the minimum thickness of an absorbing material so that it can block incoming radiation from the radioactive source. To block incoming radiation, the required material depends on the type of radiation that the user would want to block. $\alpha$ radiation is blocked by paper, $\boldsymbol{\beta}$ radiation by metals and $\gamma$ radiation by concrete and/or lead, as shown in figure 4 . This laboratory focuses on blocking $\boldsymbol{\beta}$ radiation.
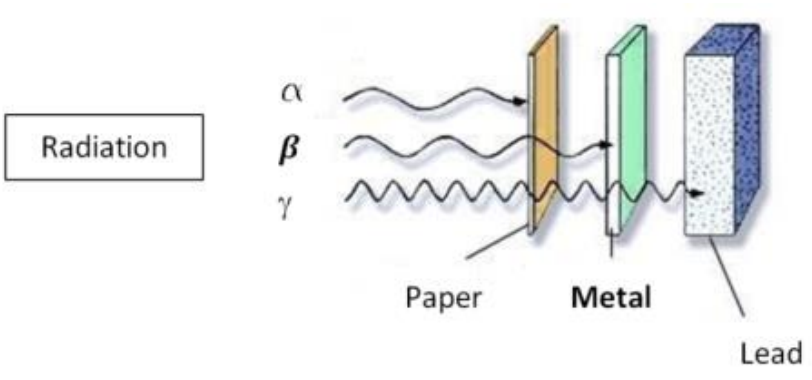

Figure 6: Radiation types and blocking materiales

In order to obtain the minimum thickness of the radiation-absorbing metals, the student will have the superficial density of the piece of metal, and the density of the metal, which is always constant. Using these data, it is possible to obtain experimentally the thickness of the absorbing metal.

On a real experiment (as shown on figure 5), the required elements include a $\boldsymbol{\beta}$ particle source, absorbers of various thicknesses, and a Geiger counter (Parks, 2001). 


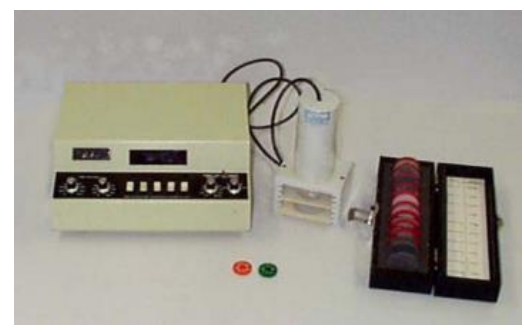

Figure 7: Real absorption experiment setup

The simulated elements are shown on figure 6 . The simulator returns activity count values corresponding to the radioactive source and the thickness and type of the absorbing material being simulated, with the radioactive source being on the third slot of the tower, and the absorbing material being on the second slot. The data obtained will be a value based on the results of a real experiment shown on table 2 .

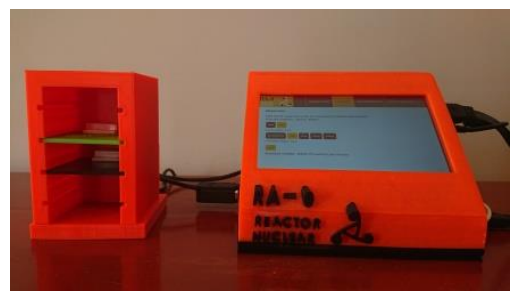

Figure 8: Simulated absorption experiment setup

The user of the simulator should take note of the activity count values displayed by the simulator. These values can then be plotted and, through and approximation technique (on most cases, minimum squares), it is possible to obtain an exponential fit to the obtained data. An example of this curve is shown on figure 7. The fit of the curve will be of the form $\boldsymbol{y}=\boldsymbol{a} \boldsymbol{e}^{-\boldsymbol{b x}}$, where the absorption coefficient can be calculated by obtaining the value of $b$ (Martinez Alonso \& Losada Ucha, 2000).

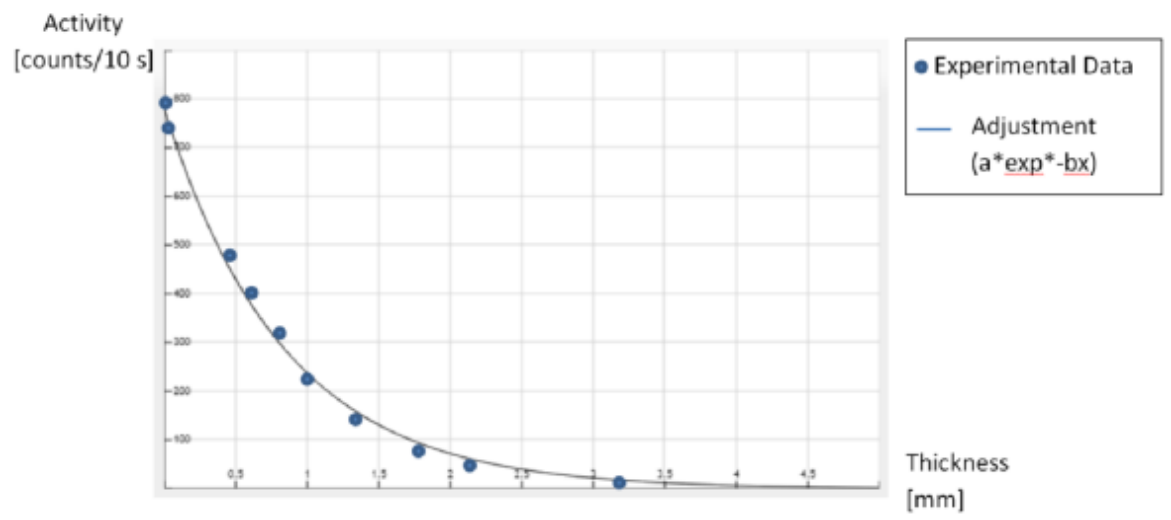

Figure 9: Simulated absorption experiment curve fitting 
The next step on the experiment is to obtain the maximum energy before $\boldsymbol{\beta}$-decay. This can be done using the thickness of the material that resulted in the lowest amount of counts per second, and then mathematically obtaining the maximum energy with this data.

Finally, comparing the maximum energy with known $\boldsymbol{\beta}$-decay patterns (as shown on figure 8 (Knoll, 2000)), the user can determine the radioactive being used.

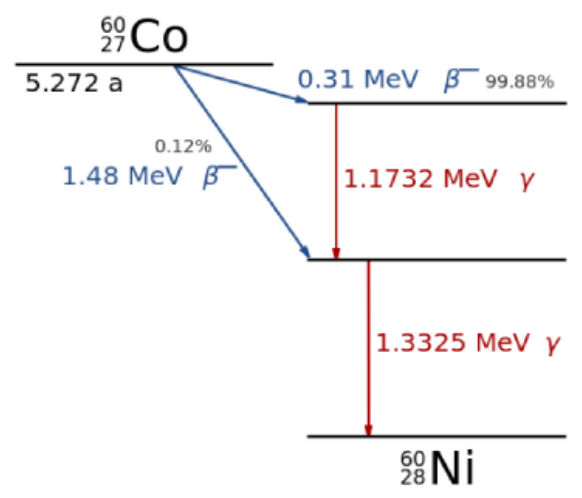

Figure 10: b-decay of Cobalt-60

\begin{tabular}{|l|c||l|c|}
\hline \multicolumn{2}{|c|}{ Co-60 Source } & \multicolumn{2}{c|}{ Na-22 Source } \\
\cline { 2 - 3 } Absorbent material & Measured activity (cpm) & Absorbent material & Measured activity (cpm) \\
\hline Without absorbent & 2861 & Without absorbent & 16962 \\
\hline Paper & 2632,12 & Paper & 15605,04 \\
\hline $45 \mathrm{mg} / \mathrm{cm}^{2}$ & 1773,82 & $45 \mathrm{mg} / \mathrm{cm}^{2}$ & 10516,44 \\
\hline $290 \mathrm{mg} / \mathrm{cm}^{2}$ & 572,2 & $290 \mathrm{mg} / \mathrm{cm}^{2}$ & 3392,4 \\
\hline $1100 \mathrm{mg} / \mathrm{cm}^{2}$ & 28,61 & $1100 \mathrm{mg} / \mathrm{cm}^{2}$ & 169,62 \\
\hline
\end{tabular}

Table 3: Activities from radiactive sources

\section{Laboratory 3: Backscattering}

This laboratory experiment analyzes the radiation from the radioactive source when the incident $\boldsymbol{\beta}$ radiation scatters when going through different materials. This experiment shows that the materials used as scattering materials exhibit this behavior, and that the scattering effect is more prominent on materials with a higher atomic number.

When a $\boldsymbol{\beta}$ particle coming from a radioactive source interacts with a material, its trajectory may deviate depending on its initial energy. This produces a dispersion effect on the particle. Depending on the relative orientation of the impact between the $\boldsymbol{\beta}$ particle and the core of the material's atoms, there is a chance that the $\boldsymbol{\beta}$ particle exits the material on the same spot where it entered the material. This phenomenon is called backscattering. 
In this experiment, the user first measures the radiation activity count when there is no scattering material in between. After that, the user adds different materials at the end of the source and measures the activity count once again. The difference between the two allows to determine which is the scattering material being used. A schematic of the experiment setup is shown figure 9 .

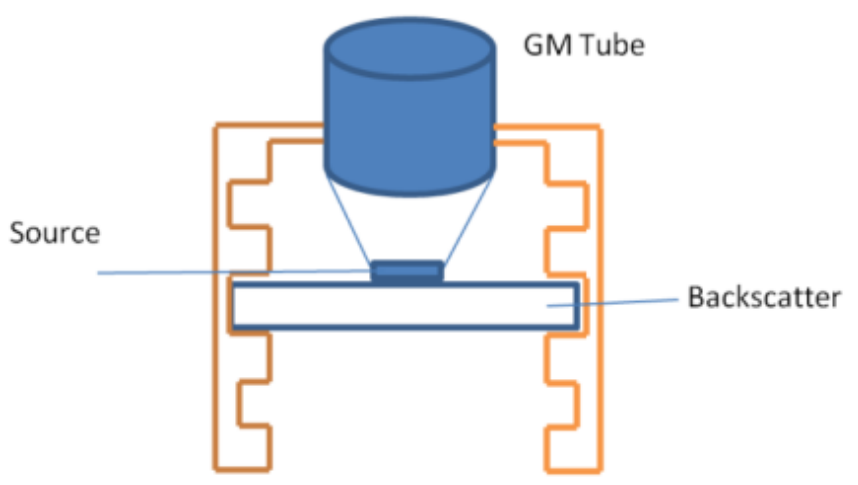

Figure 11: Backscattering experiment schematic setup

In the simulator, the setup is shown in figure 10. The different simulated elements (the radioactive source and the backscattering material) are placed in a tower similar to the one used on the previous experiment. The position of the simulated elements resembles the real experiment.

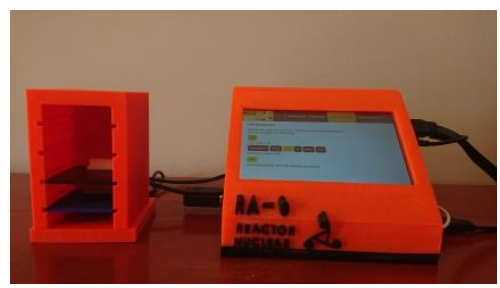

Figure 12: Backscattering experiment simulated setup

In the practical work of interaction of radiation with matter, for the study of the dispersion of charged particles, the real data shown in table 3 is stored in the simulator.

\begin{tabular}{|l|c|}
\hline \multicolumn{1}{|c|}{ Backscatter } & Average measured activity (cpm) \\
\hline Without backscatter & 2899,666 \\
\hline Aluminum & 3276,62258 \\
\hline Iron & 3537,59252 \\
\hline Zinc & 3624,5825 \\
\hline Cadmium & 3914,5491 \\
\hline Lead & 4204,5157 \\
\hline
\end{tabular}

Table 4: Backscatter 


\section{Laboratory 4: Background radiation}

This laboratory focuses on the radiation that exists on the environment, without the deliberate introduction of a radioactive source. This radiation is also called background radiation. On a laboratory setting, figure 11 , if the objective is to measure the radiation counts of a specific radioactive source, the Geiger counter will inevitably count the background radiation, the latter should be measured beforehand to appropriately subtract the background component from the measurement.

This laboratory is an expansion made to the previous iteration of the simulator, which did not have this experiment implemented (Lazarte et al., 2016).

The experiment consists in measuring the amount of counts measured by the Geiger counter over a short period of time, without any radioactive source nearby to prevent noisy data (Mera, 2015). This measurement should be done a number of times. Based on a real experiment (Suárez, n.d.), the simulated experiment simulates a measurement every 2 seconds, doing this a total of 100 times. The measured counts can be plotted in a histogram, which should resemble a Poisson distribution frequency histogram, with parameter $\lambda=1.06$ as the average amount of counts every 2 seconds, as shown on figure 12. A table 4 with the measured counts and the histogram of it are plotted on the display.

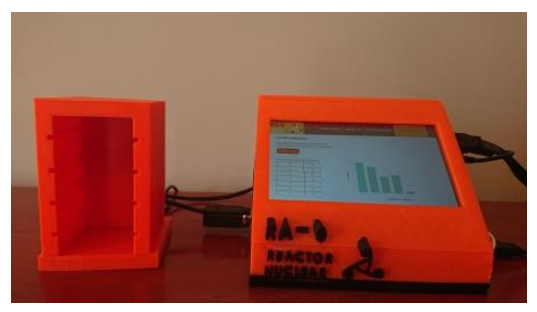

Figure 13: Setup for background radiation measurement

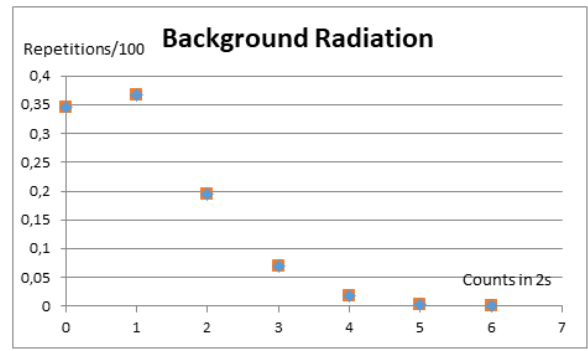

Figure 14: Background radiation

\begin{tabular}{|l|l|}
\hline Conts/100 & Value \\
\hline 0 & 0,346 \\
\hline 1 & 0,367 \\
\hline 2 & 0,195 \\
\hline 3 & 0,069 \\
\hline
\end{tabular}




\begin{tabular}{|l|l|}
\hline 4 & 0,018 \\
\hline 5 & 0,004 \\
\hline
\end{tabular}

Table 5: Radiation counts/100 values

\section{Validation and results}

The improvements made on the simulator for this iteration were developed during the COVID-19 pandemic.

As on-site lectures and laboratory work went virtual, the changes were not able to be validated with students. For this reason, this section will be based on the results obtained with the previous iteration of this simulator; which was used on late 2019, on a course of "Methodology and Application of Radionuclides", taught by the University Center for Nuclear Technology (CUTeN), of Faculty of Exact, Physical and Natural Sciences, part of the National University of Cordoba, Argentina.

The course consisted of 30 students, where as part of the laboratory practice exercises, they used the presented simulator. At the end of the corresponding exercises, the students were asked to answer a survey that focuses on the effectiveness and the overall reception of the simulator and the experiments conducted with it.

The table 5 indicates the questions asked to the students on the survey, and the figure 13 and figure 14 indicate the results obtained from the survey. The first two questions refer to information about similarity to the real experiments and understanding the real phenomena; the other three questions ask the user to rate the degree of learning that they have achieved of various concepts taught with the help of the simulator.

The results from the survey indicate that students perceived positive effects on the learning process of different topics related to the usage on the simulator, and they also showed a high degree of acceptance of the tool.

\begin{tabular}{|c|c|}
\hline Question & Options \\
\hline Q1: The simulator is similar to its real counterpart & \multirow{2}{*}{$\begin{array}{c}\text { Greatly agree, } \\
\text { Agree, } \\
\text { Slightly agree, } \\
\text { Do not agree }\end{array}$} \\
\hline $\begin{array}{l}\text { Q2: Using the simulator helped me understand the } \\
\text { physical phenomena }\end{array}$ & \\
\hline $\begin{array}{l}\text { Q3: Rate your learning achievement of the working } \\
\text { mechanisms of the instruments }\end{array}$ & \multirow{3}{*}{$\begin{array}{c}\text { Achieved, } \\
\text { Partially achieved, } \\
\text { Not achieved }\end{array}$} \\
\hline $\begin{array}{l}\text { Q4: Rate your learning achievement of radioprotection } \\
\text { (distance-time-protection) }\end{array}$ & \\
\hline $\begin{array}{l}\text { Q5: Rate your learning achievement of the behavior of } \\
\text { radionuclides (decay, half-life period) }\end{array}$ & \\
\hline
\end{tabular}

Table 6: Questions and possible answers asked on the survey 


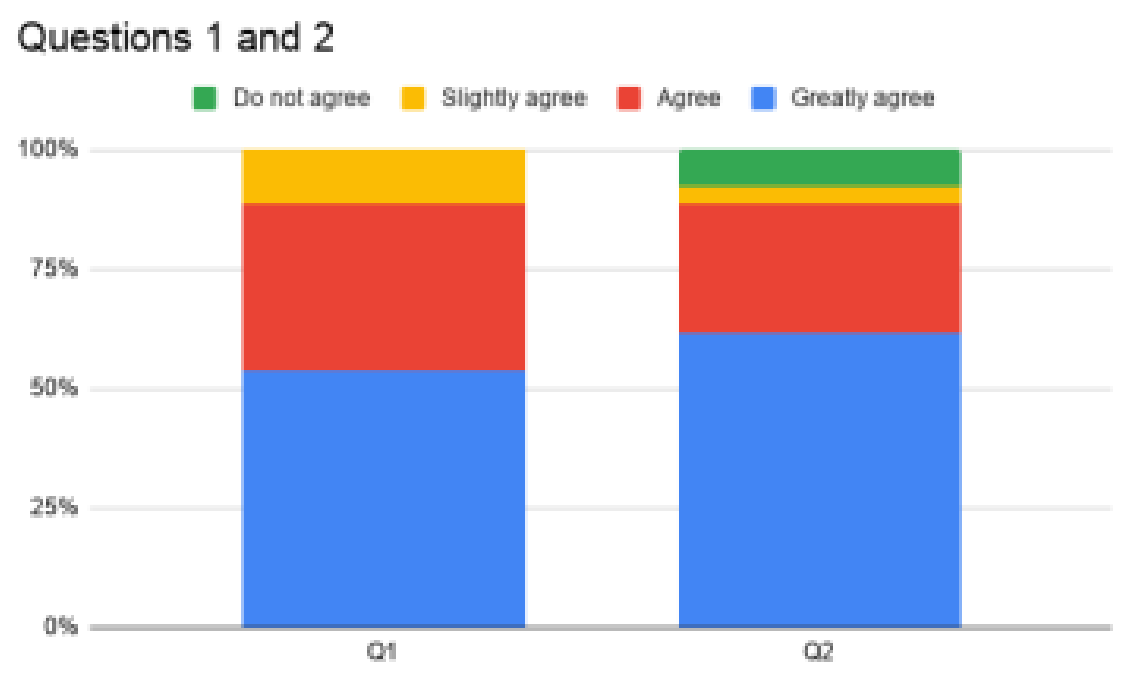

Figure 15: Answers to Questions 1 and 2

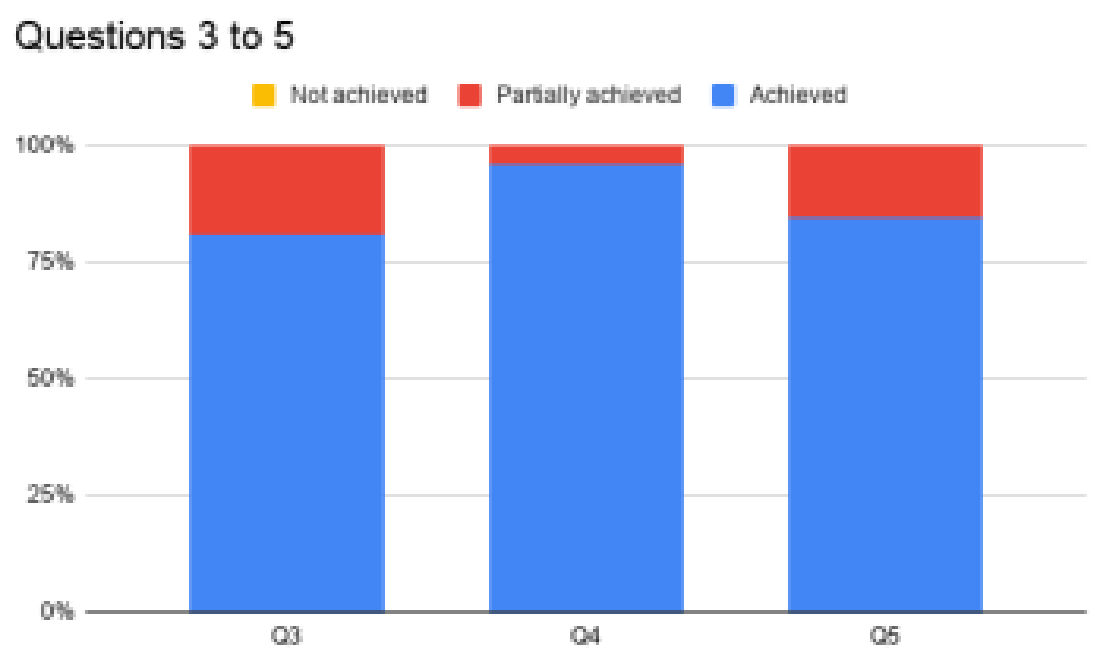

Figure 16: Answers to Questions 3 to 5

\section{Future work}

This simulator is part of a broader project. As part of the scope of the extended project, it is planned, in the near future, to upgrade the prototype of the simulator to allow usage both physically and remotely through the internet. The implementation done in this iteration can, in the future, be extended more easily than in the previous iteration to enable remote usage. 
Another feature under consideration is to add new tables for the radioactive activity values for other radioactive sources, absorbent materials and dispersers.

Finally, adding new simulations for other experiments not implemented already is also under consideration.

\section{Conclusion}

The simulator presented in this article has as its main objective to improve laboratory activities on schools, universities and laboratories. Laboratory experiments develop students' skills in dealing with laboratory instruments and physical processes with the objective of reinforcing the understanding of the investigated subject (Malkawia \& AlAraidahb, 2013).

This prototype of a simulator of a nuclear radiation counter was designed and built having as main purpose allowing to obtain real data using simulated elements. This simulator has four modes related to real-life experiments: obtaining the plateau curve that shows the basic behavior of a Geiger counter; obtaining curves that show the radioactive activity in relation to the thickness of the absorption materials which can, in turn, be used to estimate the energy of the particle-emitting source; evaluating the behaviour of radiation as it goes through dispersers; and finally, measuring the background radiation of an environment.

As the radioactive activity data provided by the simulator is based on real experiment, the usage of this simulator allows to manipulate radioactive sources, absorbing materials and particle dispersers in a safe, simulated environment that resembles the real world experiments.

This simulator is the second iteration of a broader project. This part adds usage through a touchscreen that, even though the interface deviates from actual instruments due to the lack of buttons, it makes the simulator more durable and does not compromise the validity of the data. In addition, this simulator adds the capabilities to return data based on statistical parameters in order to more closely resemble an actual experiment. Finally, this simulator is easier to extend in the future for remote usage.

The remote experiments will be introduced to students gradually in three planned phases: in the first phase, in preparation for a typical remote laboratory experiment, the students are tasked with re-familiarizing themselves with the underlying physical principles, the experimental equipment and procedure of the particular experiment to be performed. In the second phase of the laboratory experience, will use the supervision of an instructor and in the third stage, the studends will continue more detailed experimental studies in a remote fashion (Nickerson et al., 2007).

\section{References}

[1] Chautemps, N. A., Lazarte, G., \& Perez Lucero, A. L. (2019). Desarrollo de instrumentos para laboratorios remotos 
[2] de protección radiológica. Revista de enseñanza de la Física, 31 (Extra), 189194.

[3] Knoll, G. F. (2000). Radiation detection and measurement (3rd). Wiley.

[4] Lazarte, G., Perez Lucero, A., Chautemps, N. A., \& D'iaz, L. C. (2016). Simulador prototipo de contador de

[5] radiación nuclear. XI Congreso de Educación en Tecnología y Tecnología en Educación.

[6] Malkawia, S., \& Al-Araidahb, O. (2013). Students' assessment of interactive distance experimentation in nuclear

[7] reactor physics laboratory education. European Journal of Engineering Education, 38 (5), 512-518.

[8] Martinez Alonso, G., \& Losada Ucha, R. (2000). Laboratorio de alcance de radiaciones nucleares basado en

[9] microcomputadora. Ingenierías, 3 (6), 15-21.

[10] Mera, E. (2015). Experimento: Radiación. Universidad Tecnológica Metropolitana de Chile.

[11] Nickerson, J. V., Corter, J. E., Esche, S. K., \& Chassapis, C. (2007). A model for evaluating the effectiveness of

[12] remote engineering laboratories and simulations in education. Computers Education, 49, 708-725.

[13] Parks, J. E. (2001). Attenuation of radiation. Department of Physics and Astronomy, The University of Tennessee.

[14] Pérez Lucero, A., Chautemps, N. A., Bertone, R., \& Díaz, L. C. (2015). Simuladores aplicados en laboratorios de

[15] energía nuclear. XXI Congreso Argentino de Ciencias de la Computación.

[16] Suárez, P. M. (n.d.). Estudio de la radiación con un detector geiger müller. Universidad de Oviedo. 